\title{
The Tetrafigap Survey on the long-term outcome of tetraplegic spinal cord injured individuals: Part I. Protocol and methodology
}

\author{
J-F Ravaud ${ }^{1}$, J-F Désert ${ }^{2}$, D Boulongne ${ }^{2}$, M Delcey $^{3}$, J-P Pedelucq $^{4}$, M Tramblay ${ }^{5}$, A Cayot-Decharte ${ }^{3}$, A Papa ${ }^{3}$, \\ M Maury ${ }^{3}$ and the Tetrafigap Group* \\ ${ }^{1}$ INSERM (National Institute for Health and Medical Research), IFRH (Federative Research Institute on \\ Disability); ${ }^{2}$ Centre of Neurological Rehabilitation, Coubert; ${ }^{3}$ Association des Paralysés de France $\left(\right.$ APF); ${ }^{4}$ Centre \\ Mutualiste de rééducation et de réadaptation fonctionnelle, Kerpape; ${ }^{5}$ Rehabilitation Department, Hospital Raymond \\ Poincaré, Garches, France
}

The Tetrafigap Survey, a multicentre epidemiological survey on the outcome of tetraplegic spinal cord injured (TSCI) people from their first admission to a Rehabilitation Department or Centre is currently being undertaken in France.

The general objective of this survey is to evaluate the situation of the TSCI people and their conditions of life in its medical, psychological and social aspects.

This first article is aimed at presenting the protocol and the methodology of this survey. In a second part, yet to be submitted for publication, the preliminary results will be presented. It was first necessary to create a database of the population of TSCI people known to the centres and medical rehabilitation services, removing double entries.

The criteria used for inclusion in the study were: a complete or incomplete traumatic cervical cord lesion, including post-surgical complications; age 16 or over at the time of the accident which must have occurred before December 31, 1992.

The enquiry consisted of a self-administered questionnaire carried out with surviving tetraplegic people who had given their informed consent for their participation. The questionnaire consecutively covered the following topics: the situation at the time of the accident, the medical evolution between the accident and the end of stay in a rehabilitation unit, their evolution after discharge and the current situation (medical, social, professional and personal).

During this first phase, 6082 TSCI people were identified by the collaborating centres. The 603 files of those who had died and 769 double entries were removed. Thus, 4710 questionnaires were sent out. The results of the participation show that 2251 people gave their consent and received questionnaires (340 additional deaths were acknowledged at this step), 163 refused, 869 were lost for follow-up, and 67 were excluded. There was no reply from 1020 people. We received 1830 questionnaires of which 1669 fulfilled all the necessary criteria for data exploitation. Home interviews with people who gave their consent will be carried out in a second phase as well as a study of deaths. A 5-year longitudinal follow-up is scheduled.

Keywords: spinal cord injury; tetraplegia; epidemiology; long-term outcome; medical followup; life conditions

Correspondence: J-F Ravaud MD, CERMES-INSERM U.304, 182
Boulevard de la Villette, F75019, France
*The Tetrafigap Survey, carried out under the aegis of the
'Association Francophone Internationale des Groupes d'Animation
de la Paraplégie' (AFIGAP) was performed thanks to the
participation of the Tetrafigap group which involves the following
35 associated teams: (1) Berck-Hôpital franco-américain (Dr Allas);
(2) Berck-Centre Hélio-marin (Dr Chilkhi); (3) Bichat (Pr Bedoiseau);
(4) Bois-Guillaume (Pr Beuret-Blanquart); (5) Bordeaux (Pr Barat,
Dr Petit); (6) Bouffémont (Dr Thévenin-Lemoine); (7) Bourbon-
Lancy (Drs Malier, Esteve); (8) Bruxelles (Drs Heilporn, Bienfot); (9)
Cerbère (Dr Enjalbert, Rosebande); (10) Charleville Mézières (Drs
Delrez, Skoda); (11) Club de loisirs et d'entraide de Garches; (12)
Coubert (Dr Désert); (13) Garches (Prs Bussel, et Dizien, Drs Lortat-
Jacob Tramblay); (14) Genève-Hôpital Cantonal Universitaire, (Pr
Chantraine Dr Castagnier); (15) Giens (Drs Boucand) Tournebise);
(16) Invalides (INI, Drs Dumurgier, Barouti); (17) Kerpape (Drs Busnel, Cormerais, Pédelucq); (18) La Membrolle sur Choisille (Dr Chauvet); (19) L'Arche (Drs Egon, Isambert); (20) La Tour-deGassies (Drs N'Guyen Van Tam, Wiart); (21) Lay-Saint-Christophe (Drs Brugerolle, Thisse); (22) L'Hospitalet-Montoire (Dr Simon); (23) Limoges (Prs Labrousse et Dudognon, Dr Munoz); (24) LyonHenry Gabrielle (Pr Boisson); (25) Lyon-Henry Gabrielle (Pr Eyssette, Dr Frenay); (26) Marseille-Clinique St-Martin (Dr Chapuis-Ducoffre); (27) Marseille-Valmante (Drs Benezet et Allovon); (28) Montpellier-Propara (Drs O'Hanna, Rouays-Mabit); (29) Mulhouse (Dr Jacob-Chia); (30) Nantes (Pr Mathé, Drs Perrouin-Verbe, Rome); (31) Neufmoutiers-en-Brie-Les Lycéens (Dr François); (32) Pen-Bron (Dr Tanguy); (33) Rennes Beaulieu (Pr Brissot, Dr Gallien); (34) Saint-Hilaire du Touvet (Dr Pauget); (35) Strasbourg (Dr Pinelli) 


\section{Introduction}

As a result of modern resuscitation and emergency medicine, an increasing number of people presenting with traumatic cervical cord injury survive, even with high-level lesions. In addition, the long-term survival of these people has also increased since World War II. Further, the current socio-economic climate imposes on health providers the need for a rigorous control on health expenditures generated by the various pathologies.

However, there is currently no precise data on the long-term outcome of the overall spinal cord injured people, particularly for those who are tetraplegic. For this reason, the 'Association Francophone Internationale des Groupes d'Animation de la Paraplégie' (AFIGAP), the International French-Speaking Association of Animation Groups of Paraplegia has decided to undertake a multicentre epidemiological survey on the outcome of the tetra-plegic spinal cord injured people (TSCI). This survey, named TETRAFIGAP, has solicited all the centres and rehabilitation units in France and two others in European Frenchspeaking countries (Belgium and Switzerland).

Its aim is to evaluate the medical situation and the conditions of life of TSCI people. The overall protocol was launched in 1995. In 1998, a synthesis of the overall research works conducted about the selfadministered questionnaire will become available.

The first article, Part I, is aimed at presenting the protocol and the methodology of the Tetrafigap Survey. In a second part, to be submitted for publication, the preliminary results will be described.

\section{Background and literature}

Few studies have been carried out, either in Europe or elsewhere in the world, on the problems encountered by tetraplegic people after leaving rehabilitation units. Moreover, these studies deal mainly with spinal cord injured (SCI) people in general (both paraplegic and tetraplegic subjects).

At the end of World War II, Guttmann ${ }^{1}$ was one of the first authors to become interested in the specific problems of the SCI patients and their outcome.
The main studies, in this area, have been carried out in North America. Such as those of Tator et $a l^{2}$ who studied the evolution of the outcome of SCI people in the Toronto area in Canada. Two groups were studied, comparing neurological recovery, mortality and length of stay during the first hospitalisation of a group of 201 patients treated between 1974 and 1981 in a specialised Spinal Cord Injuries Unit, with a group of 351 paraplegic or tetraplegic people who had been treated in a general hospital between 1947 and 1973 (ie before the creation of the specialised unit in Toronto).

In the United States two large cohorts have been studied:

(1) DeVivo et $a l^{3}$ have collected a database which in 1991 included 13763 patients, of which 54.2\% were TSCI patients. This database, located at the National Spinal Cord Injuries Statistical Centre (NSCISC) permitted an annual follow-up of this population from its constitution in 1973, as SCI people were being included in the survey. This cohort, which is, without any doubt, the most important (cf. Table 1), has led to many publications. $^{3-5,25}$

(2) Krause $^{6}$ studied the outcome over 15 years of 135 SCI people who in 1974 had a minimum age of 18 and a 2-year follow-up period after the accident. He used a questionnaire and compared the responses obtained in 1974 with those obtained using the same questionnaire during a new interrogation of the same population in 1989. This follow-up study showed that rehabilitation of the disability continues long after the departure from the specialised centre.

There are numerous other world-wide studies on the epidemiology of SCI patients, in particular the studies by Chen and Lien in Taiwan, ${ }^{7}$ Soopramanien ${ }^{8}$ in Romania, Hart and Williams ${ }^{9}$ in South Africa, Shingu et $a l^{10}$ in Japan, Silberstein and Rabinovich in Russia $^{11}$ and Pajareya in Thailand. ${ }^{12}$ They basically deal with the causes of the accidents and the sociodemographic characteristics of the wounded people but not their outcome.

Table 1 Comparison between different surveys in the literature and the Tetrafigap Survey

\begin{tabular}{|c|c|c|c|c|}
\hline Authors & $\begin{array}{c}\text { Period of } \\
\text { study }\end{array}$ & $\begin{array}{c}\text { Year of } \\
\text { publication }\end{array}$ & Region & $\begin{array}{c}\text { Number of } \\
\text { tetraplegic } \\
\text { subjects }\end{array}$ \\
\hline DeVivo et al & $1973-85$ & 1992 & USA & 7460 \\
\hline Shingu et al & $1990-92$ & 1995 & Japan & 7317 \\
\hline Hartkopp et al & $1953-90$ & 1997 & Denmark & 424 \\
\hline Chen and Lien & $1978-81$ & 1985 & Taïwan & 262 \\
\hline Levi et al & $1991-94$ & 1995 & Sweden (Stockholm) & 147 \\
\hline Minaire et al & $1970-75$ & 1978 & France (Rhône-Alpes) & 142 \\
\hline Daverat et al & $1982-85$ & 1995 & France (Bordeaux) & 99 \\
\hline Krause & $1974-89$ & 1992 & USA (Atlanta) & 91 \\
\hline TETRAFIGAP & $1995-96$ & 1997 & France (Belgium, Switzerland) & 1669 \\
\hline
\end{tabular}


More specifically in Europe, one of the main works is a study by Levi et $a l^{13}$ of patients in the Stockholm area, Sweden, between November 1991 and June 1994. The studied population included 355 patients of whom only 147 had a cervical lesion. The data were collected by semi-directed interviews. In Denmark, Hartkopp et $a l^{14}$ studied the evaluation of the outcome and the cause of death after a SCI. They studied 888 SCI people between 1953 and 1990. Two populations were compared: the first one, was of 359 patients treated between 1953 and 1971 and the second one, of 529 patients treated between 1972 and 1990. Whiteneck et $a l^{15}$ and Frankel et $a l^{16}$ studied mortality, health condition and functional and psycho-social outcome in 1834 patients with SCI for over 20 years, who were treated in Stoke Mandeville Hospital, in the Spinal Unit in Southport in England, and in the Craig Rehabilitation Centre, in Colorado, USA.

With regard to France, the first comprehensive enquiry about the return to professional and social life of paraplegic and tetraplegic individuals was made by Maury ${ }^{17}$ as early as 1964 . Minaire et al, ${ }^{18,19}$ for the first time in France, made an extensive register of SCI patients in the Rhône-Alpes region between 1970 and 1975, thus studying the incidence and prevalence of these pathologies. But, in France and in Frenchspeaking countries, data on the outcome of SCI patients are scanty. This has been emphasised by Tricot $^{20}$ and by Yelnik et $a l^{21}$. Daverat et $a l^{22,23}$ in Bordeaux, made a cohort of 188 SCI patients admitted to the Pellegrin Hospital between 1982 and 1985. They published a study on the long-term outcome of 149 SCI patients still alive in 1992. ${ }^{22}$ In addition, they studied the predictive factors of autonomy in the 99 tetraplegic people in this group. ${ }^{23}$ Finally, Dollfus ${ }^{24}$ surveyed the state of rehabilitation of SCI patients from information collected from specialised centres in ten European countries.

These epidemiological investigations studied the prevalence and incidence of SCI and the risk factors rather than their consequences. In fact, few teams have worked on the outcome of tetraplegic people, particularly the medical, psychological and socioeconomic aspects of their daily lives.

\section{Methodology}

A Steering Committee of seven members from the fields of rehabilitation, disability movement and research was set up. This work was placed under the aegis of the AFIGAP, an association with a membership of over 200 physicians in physical medicine and rehabilitation as well as paramedical workers belonging to hospitals or rehabilitation units dealing with SCI.

The Steering Committee held regular sessions from September 1994. Its objectives are as follows:

- protocol elaboration and methodology of the survey;
- search for funding and the required collaborations;

- exploitation and follow-up of collected data.

The Survey is named TETRAFIGAP and is protected by a charter.

Criteria for inclusion into the study were:

(1) traumatic tetraplegia, complete or incomplete, including post-surgical;

(2) with or without brain injury;

(3) admission to a rehabilitation unit since its creation, whatever the duration of the stay, and even those who died following admission;

(4) age 16, or over, at the time of the accident which must have occurred before December 31, 1992, Therefore they were all adult patients at the time of the survey. All the cervical cord lesions occurred at least 2 years prior to the survey.

The overall protocol accords with the recommendations covering public's protection with respect to confidentiality. A favourable notification was given to the protocol by the 'Commission Nationale Informatique et Liberte' (CNIL), the French National Committee on Data Processing and Privacy.

Thirty-five departments or units specialised in SCI agreed to participate in the survey. Each sent a list of the TSCI patients in their service who fulfilled the inclusion criteria as well as a list of those who had died. The TSCI patients being followed-up at more than one centre were registered at the centre they had used most recently.

After eliminating the double entries and the deaths, this procedure established for each specialised unit the list of all the selected TSCI patients. A consent form was then sent to the patient, together with a letter signed by the Secretary of AFIGAP and the physician from the unit. The request was mailed again to the non-responders after 3 months.

There were five categories of replies:

- acceptance;

- refusal;

- death;

- return to sender: 'no longer at this address';

- no response.

At the same time, the questionnaire was designed by the Steering Committee. This questionnaire was sent from the centres to the patients agreeing to participate in the study. Upon completion, the replies were sent by pre-stamped mail to the Coubert Centre, the AFIGAP base.

All the questionnaires were made anonymous by a coding system which allowed a selection for a second request in case of no response before being definitively entered in the database. The material was a selfadministered questionnaire entitled '1995 Survey on the Outcome of Tetraplegic People from their First Admission either in a Department or a Rehabilitation Centre'. It comprises eight pages with 119 simple or 
multiple choice and 18 open questions, and consists of four parts:

(1) Situation at the time of the accident: family and professional situation, type of accident;

(2) Evolution between the accident and the end of stay in a rehabilitation unit: medical data (lesion level), complications (tracheostomy, bedsores, fever etc.);
(3) Outcome since the conclusion of rehabilitation: centres attended, rehospitalisations and reasons for these;

(4) Current situation: family and medical status, treatment, care needed, functional independence, technical aids, housing, social relations, external transport arrangements, current professional situa-

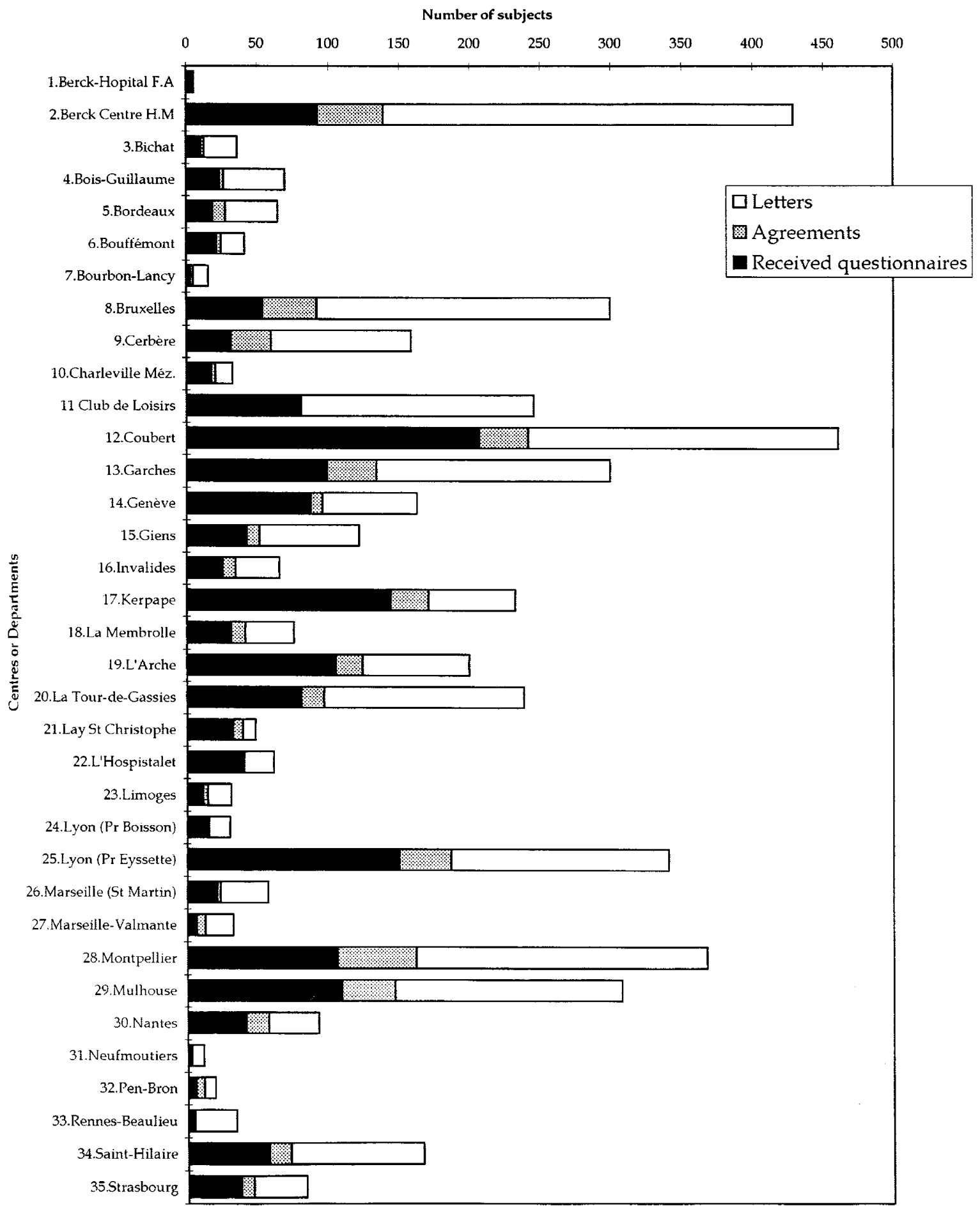

Figure 1 Contribution of the 35 centres to the Tetrafigap Survey. 
tion, training, leisure, assessment of well-being and financial resources.

At the end of the questionnaire patients were asked whether they would agree to participate in a further enquiry by an additional questionnaire or by receiving a visit from a home researcher. Finally, the patients were invited to provide precise details about their current situation and to give suggestions.

The usable questionnaires were entered and were statistically analysed (SPSS Software). Before processing, all the open questions were coded into formats specially created for this purpose. Data entering was made using the database 'ACCESS' with the help of a specific mask allowing control of as many errors as possible by rejecting erroneous or incoherent data entries. Logical verifications were carried out. The gathering and coding of the questionnaires was finished by the end of 1996 .

\section{Results of participation to the survey}

Thirty-five departments or specialised centres participated: 33 in France, one in Belgium (Brussels) and one in Switzerland (Geneva) (Figures 1 and 4).

The survey gives a good distribution throughout France with 15 of the 21 regions being represented, ie almost three quarters. The six non represented regions lack SCI specialised centres. The largest series of patients came from the Ile-de-France region where six centres participated, even although the oldest centre (Garches) could not use all of its archives. It seemed logical to include the Brussels and Geneva Centres as their populations are similar and their physicians have collaborated from the beginning of the work of AFIGAP. All of the SCI centres solicited responded willingly to the request of the Steering Committee.

It is interesting to analyze the double entries because they provide a good idea of the stability of the follow-up of the TSCI subjects; the centres with the largest number were the largest and often the oldest ones. Amongst them: Berck-Hélio Marin, Coubert (former centre of Fontainebleau), Garches, Lyon-Henry Gabrielle, Kerpape, Montpellier, and in particular Cerbère where nearly half the TSCI patients were double entries.

During the first phase of the protocol, 6082 TSCI people were identified by the contributing centres. After the elimination of 769 double entries and 603 patients known to be dead, 4710 people were sent the request to participate (Figure 2). Of these, 2251 gave consent (ie 48\%), 163 refused, 340 were notified as dead by the family or neighbours, a fact that was unknown by the centres.

There were 869 'return to sender' letters as 'no longer at this address' and 1020 did not reply. Finally, 67 patients were discarded as not fulfilling the inclusion criteria (Figure 3).

Of the 2251 people agreeing to participate, 1749 returned the questionnaire after completion, ie a $77.7 \%$ reply rate. Moreover, an additional series of 81 patients,

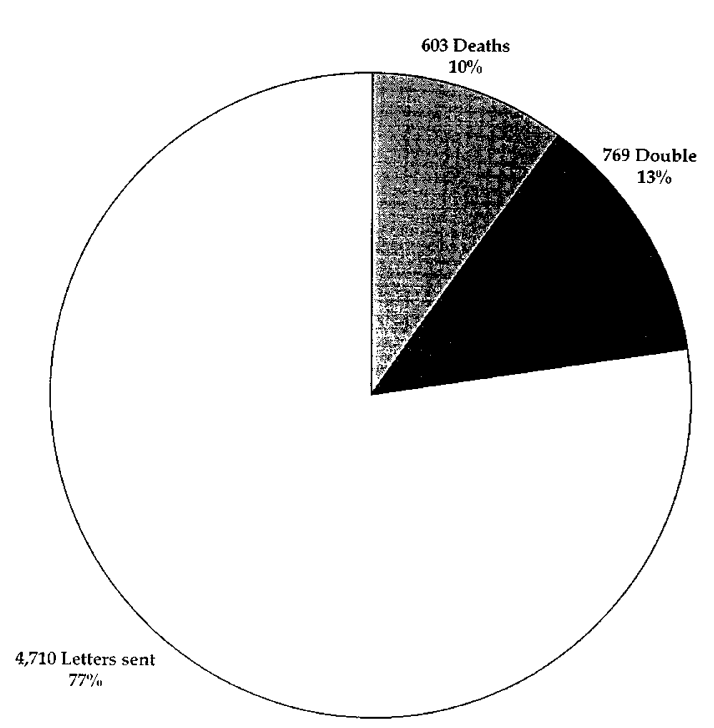

Figure 2 Recruitment of the tetraplegic population by the centres $(n=6082)$.

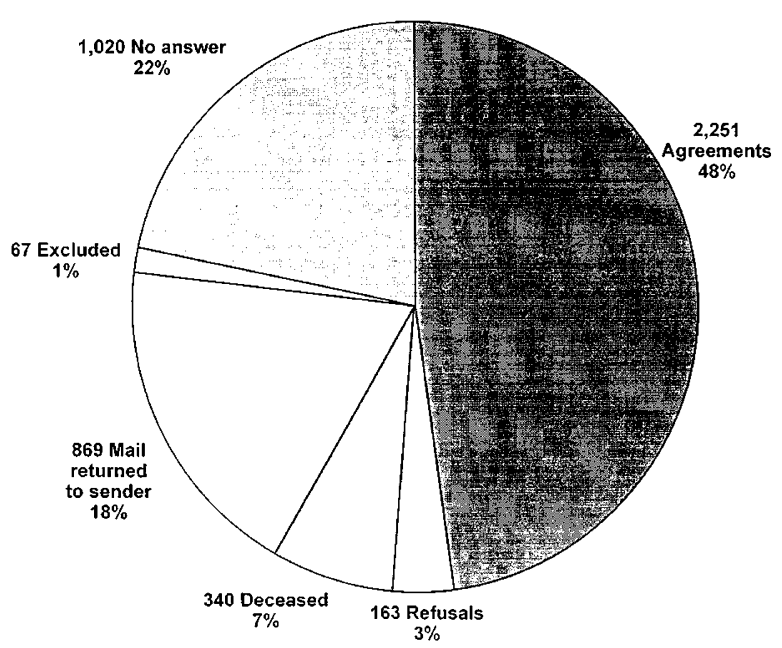

Figure 3 Participation to the Tetrafigap Survey of the tetraplegic people contacted by mail $(n=4710)$.

who were not on the files or who had been lost to followup by the centres, was provided by the TSCI people participating in the activities of the Leisure and Aid Club at Garches. Of these 1830 questionnaires $(1749+81), 161$ could not be exploited for the following reasons: death mentioned by the family on return of the questionnaire ( 7 cases); erroneous or dubious diagnoses (61 cases); age less than 16 at the time of accident (58 cases); date of accident after December 31, 1992 (35 cases).

Consequently, the analyses will only deal with 1669 effectively usable questionnaires which will constitute the final basic population of the TETRAFIGAP survey.

The actual participation rates (questionnaire returned) were very different in each centre (Figure 4) 


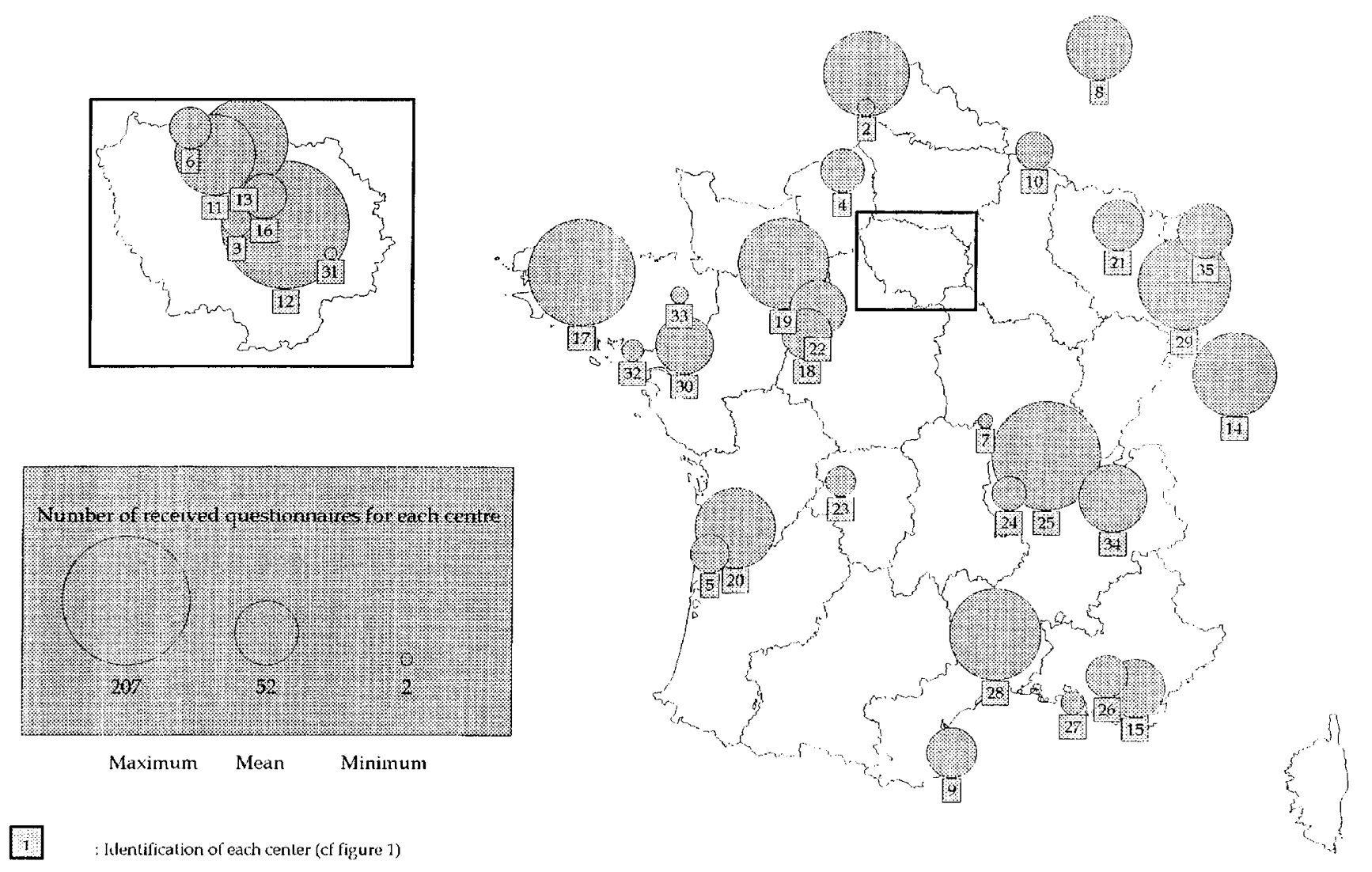

Figure 4 Geographical distribution of participation of the 35 rehabilitation centres to the Tetrafigap Survey.

but the distribution was fairly good as the centre with the highest number of returns of questionnaires only represented $12 \%$ of the total.

\section{Discussion}

The national and even the international character of the survey must be emphasised. Most enquiries already carried out were often regional. The Tetrafigap Survey has allowed the collection of a large population of specifically TSCI people, rather than a population of SCI patients in general as is the case with most of the previously mentioned studies.

However, this study is not really a census of TSCI patients and even although it is a national study it does not pretend to be exhaustive. Its main aim was to obtain a sample representing as faithfully as possible the TSCI population, in particular reflecting their wide geographical distribution. Thus, it was not a study of prevalence. In the protocol, children, those with recent traumas, and those with non-traumatic tetraplegia were excluded. In addition, as the selection method was based on 35 rehabilitation centres, it did not take into account tetraplegic people who were cared for outwith these centres or abroad.

The features of the participation in the survey merit certain comments. Only $48 \%$ of the 4710 patients who received a participation request gave their consent
(Figure 3). The true percentage is probably higher as it is likely that a large number of deaths were not identified among the non responders on the 'address unknown' groups. If only the living patients giving approval or refusing are taken into account, a figure of 2201 TSCI people out of a population of 60 million would be reached. Putting this figure of 38 per million into perspective with the prevalence figures by Minaire et $a l^{18}$ who found a rate of 88 per million and by Levi et $\mathrm{al}^{13}$ with a rate of 93 per million, the coverage of our survey would be approximately half of French people who are tetraplegic. However, we should take note that the North American studies showed much higher prevalences: 491 per million by DeVivo et $a l^{25}$ in 1980 and 290 per million by Griffin et $a l^{26}$, if we assume a figure of $50 \%$ with cervical lesions among the total of SCI patients (the average found in the world literature mentioned above). In Europe these figures do not seem so high.

The uniqueness, as well as the major problem of this survey, comes from the difficulty in coordinating the work of 35 centres with different staffing levels and the numbers of files for processing being quite different between centres (Figure 1).

In any case, this approach represents a scientific collaboration unprecedented in the French-speaking field of rehabilitation, which was made possible thanks to the AFIGAP network. 
The rate of return of questionnaires $(77.7 \%)$ is satisfactory considering:

(1) the length of the procedure, in particular between the first agreements (July 1995) and the sending out of questionnaires (from November 1985 to January 1996);

(2) the number of immigrants with linguistic difficulties and the inadequacy of some psychosocial items of the questionnaire because of some cultural particularisms;

(3) the length of the questionnaire (137 questions) and the time required for its completion.

This self-administered questionnaire allowed, as far as was possible, the gathering of the points of view of people regarding their own personal situation. The quality of the medical information was checked after collection and logical verifications allowed a few corrections. This relatively long questionnaire has the major advantage of simultaneously dealing with medical, functional, psychological and social aspects. The main objective of the survey was to assess the long-term outcome and therefore the living conditions on departure from the rehabilitation centres. The TSCI people were asked about their family, professional, social and financial status. These aspects are in fact very poorly known, and this survey should permit a better understanding of these essential data, in particular to promote a fairer compensation for injury. We expect that with an additional home survey, we will be able to refine this information in more detail.

The 950 TSCI patients who died were recorded at various phases of the survey -603 by the centres, 340 as a result of the request for participation and seven by the families after the questionnaire had been sent in 1995 or 1996.

Currently, the cause of death is only known in about $40 \%$ of cases and is less well known when death occurred outside a centre. These data on deaths will be simultaneously exploited in a specific fashion. A systematic search for the causes of death still unknown as well as the number of deceased patients among those lost to follow-up will be undertaken.

This analysis of mortality data has a major importance for us as we do not know with any accuracy the causes of death in TSCI patients as well as their link with tetraplegia. We will study the evolution of survival duration since the creation of the centres with respect to age at the time of the accident, and the causes of mortality in comparison with those observed by De Vivo. ${ }^{5}$

\section{Perspectives}

This survey is scheduled in three phases: Phase 1, whose methodology is described in this article. Statistical analysis of the replies to the questionnaire are the object of different works targeted at specific topics. Research workers and residents undertaking their medical theses are currently working on the analysis of psychosocial aspects, medical complications, functional abilities, dependency and socioprofessional reinsertion; Phase 2, which is qualitative, will consist of a more thorough analysis of the initial survey thanks to interviews of TSCI people who agreed for a home visit. Of the 1669 people with a usable questionnaire, 1495 accepted the request to complete a further questionnaire and 1125 accepted a home interview. Therefore, it is planned to prepare an interview and clinical examination grid and to set up a sample among people who gave their consent; and Phase 3 would allow for a longitudinal follow-up currently planned at 5 years by a requestioning of the population participating in Phase 1 and by the inclusion of all new cases of TSCI.

\section{Acknowledgements}

The TETRAFIGAP Survey was carried out with grants from the following organisations which we wish to thank: HANDITEC, the 'Association de Gestion du Fonds pour l'Insertion Professionnelle des Personnes Handicapées' (AGEFIPH), the 'Association des Paralysés de France' (APF), the 'Fondation des Mutuelles du Mans' (Fondation de France), the 'Fondation Suisse pour la Paraplégie', the 'Centre Européen d'Etudes de Sécurité et d'Analyses des Risques' (CEESAR), the 'Institut pour la Recherche sur la Moelle Epinière' (IRME).

\section{References}

1 Guttmann L. Spinal Cord Injuries: Comprehensive Management and Research. 2nd edn. Blackwell Scientific Publications: Oxford 1976.

2 Tator $\mathrm{CH}$ et al. Neurological recovery, mortality and length of stay after acute spinal cord injury associated with changes in management. Paraplegia 1995; 33: 254-262.

3 DeVivo MJ, Richards JS, Stover SL, Go BK. Spinal Cord Injury: Rehabilitation Adds Life to Years. West J Med 1991; 154: $602-$ 606.

4 DeVivo MJ, Richards JS. Community reintegration and quality of life following spinal cord injury. Paraplegia 1992; 30: $108-$ 112.

5 DeVivo MJ, Black KJ, Stover SL. Causes of death during the first 12 years after spinal cord injury. Arch Phys Med Rehabil 1993; 74: $248-254$.

6 Krause JS. Longitudinal Changes in Adjustment After Spinal Cord Injury: A 15-Year Study. Arch Phys Med Rehabil 1992; 73: $564-569$.

7 Chen CF, Lien IN. Spinal Cord Injuries in Tapei, Taiwan, 1978 1981. Paraplegia 1985; 23: $364-370$.

8 Soopramanien A. Epidemiology of spinal injuries in Romania. Paraplegia 1994; 32: 715-722.

9 Hart C, Williams E. Epidemiology of spinal cord injuries: a reflection of changes in South African Society. Paraplegia 1994; 32: $709-714$.

10 Shingu $\mathrm{H}$ et al. A nationwide epidemiological survey of spinal cord injuries in Japan from January 1990 to December 1992. Paraplegia 1995; 33: $183-188$.

11 Silberstein B, Rabinovich S. Epidemiology of spinal cord injuries in Novosibirsk, Russia. Paraplegia 1995; 33: 322 - 325.

12 Pajareya K. Traumatic spinal cord injuries in Thaïland: an epidemiologic study in Siriraj Hospital, 1989-1994. Spinal Cord 1996; 34: 608-610.

13 Levi R, Hulting C, Nash MS, Seiger A. The Stockholm spinal cord injury study: 1. Medical problems in a regional SCI population. Paraplegia 1995; 33: $308-315$. 
14 Hartkopp A, Bronnum-Hansen H, Seidenschnur AM, BieringSorensen F. Survival and cause of death after traumatic spina cord injury. A long-term epidemiological survey from Denmark. Spinal Cord 1997; 35: 76-85.

15 Whiteneck GG et al. Mortality, morbity and psychosocial outcomes of persons spinal cord injured more than 20 years ago. Paraplegia 1992; 30: 617-630.

16 Frankel HL et al. Changes in long-term survival in spinal cord injury: a fifty year investigation. Abstract published in the book of abstracts of the 35th Annual Scientific Meeting of IMSOP: Atlanta, Georgia, USA, August 1996; pp 13.

17 Maury M. Readaptation des travailleurs porteurs de lésions de la colonne vertébrale et de la moelle épinière. Réadaptation 1964; 107: $19-30$.

18 Minaire $\mathrm{P}$ et al. Epidemiology of spinal cord injury in the RhôneAlpes region. Paraplegia 1978-79; 16: $76-87$.

19 Minaire $\mathbf{P}$ et al. Life expectancy following spinal cord injury: a ten year survey in the Rhône Alpes region, France, 1969-1980. Paraplegia 1983; 21: $11-15$

20 Tricot A. Etiologie et épidémiologie des lésions médullaires. In Maury M. (ed) La paraplégie chez l'adulte et l'enfant. Flammarion Médecine-Sciences Paris 1981: 1-14.
21 Yelnik A, Dizien O, Cordier C, Held JP. Epidémiologie des paraplégies traumatiques. Quelques problèmes méthodologiques. In Rencontres autour du blessé médullaire. Masson Paris 1990: $191-196$

22 Daverat $\mathrm{P}$ et al. The long term outcome in 149 patients with spinal cord injury. Paraplegia 1995; 33: 665-668.

23 Daverat P, Dartigues JF, Mazaux JM, Barat M. Etude prédictive de l'autonomie chez le tétraplégique post-traumatique. Ann Réadap Méd Phys 1990; 33: 461 - 468.

24 Dollfus P. Paraplégie: La situation de la réadaptation à l'heure européenne. In Rencontres autour du blessé médullaire. Masson Paris 1990: $191-196$.

25 DeVivo MJ, Fine PR, Maetz HM, Stover SL. Prevalence of spinal cord injury. A re-estimation employing life table techniques. Arch Neurol 1980; 37: 707-708.

26 Griffin MR et al. Traumatic spinal cord injury in Olmsted County Minnesota, 1935-1981. Am J Epidemiol 1985; 121: 884-895. 